\title{
Condition analysis of a multicopter carried with passive skid for rough terrain landing
}

\author{
Maozheng Xu, Taku Senoo and Takeshi Takaki
}

\begin{abstract}
This paper describes the condition analysis of a multicopter carried with a proposed device for rough terrain landing. Based on a multicopter carried with an electrical robot arm for grasping, we proposed a method to determine whether the skid-carried multicopter can land on an arbitrary slope or not. We established the static model of the entire device, and analyzed the conditions under which the arm and skid can contact the arbitrary plane and the COG (Center of Gravity), which includes the mass of passive skid, multicopter body and each link of the robot arm. Further, we proposed a method to analyze whether the entire device can land stably. By analyzing that the projection of the entire device's COG is inside or outside the triangle, that comprises the contact point between the device and the uneven ground, we can determine whether the device can land successfully and the condition for capable landing is concluded. After the numerical analysis, the verification experiment is conducted, and by comparing the result of analysis with the experiment, the accuracy of the analysis can be demonstrated.
\end{abstract}

Keywords: Landing, Passive skid, Rough terrain

\section{Introduction}

Owing to the outstanding advantages such as light weight and low cost, multicopters are now utilized frequently in fields. Occasionally, multicopters perform tasks that humans cannot realize with high precisions, such as rescue, agriculture, inspection [1,2], and express delivery [3], etc. Although people are enjoying the innumerable conveniences that multicopters provide, more researchers are beginning to realize the importance of landing condition to the multicopter. Suitable landing conditions can be important, especially in several fields, such as inspection and express delivery, otherwise the multicopter may crash, and cause mission failure. As an essential part of landing, the condition of the landing site is also critical, among other factors. Generally, for multicopters, a flat ground is the best condition for landing when conducting missions. However, landing on flat ground is only in ideal situations. In an environment where severe

*Correspondence: takaki@hiroshima-u.ac.jp

Hiroshima University, Hiroshima, 1-4-1 Kagamiyama, Higashi-Hiroshima, Hiroshima 739-8527, Japan disasters, such as earthquakes, mudslides, or tsunamis have occurred, the terrain becomes irregular, and even contains numerous obstacles such as rocks or broken debris. In these cases, a multicopter that can land on uneven ground or rough terrain is required.

Generally, research on uneven ground mobile robots, including multicopters, can be divided into three categories: research on control system, research on obstacle avoidance, and research on mechanism design for perching or landing on uneven ground. To control robots moving on uneven ground, researchers from Easwari Engineering College monitored the landing mechanism of the multicopter, by using gyroscope and accelerometer [4], and researchers from The University of Tokyo proposed a vision-based autonomous landing system for a fixed wing multicopter [5]. In contrast, lots of researchers have focused on the development of a control system for multicopters, to achieve a better result, not solely for landing, but also for action synchronization of the mechanism [6-9]. For uneven ground or rough terrains, a number of researchers have focused on obstacle avoidance mechanism, such as stair-climbing robots [10-14], 
wall-climbing devices [15], and even mobile robots with semicircular wheels [16]. Considering that solely moving on uneven ground is inadequate, more researchers are beginning to focus on the mechanical structure that can make the unmanned aerial vehicle (UAV) land steady.

Landing refers to a series of processes from the moving state of the flying object in the air, to contact with the ground, and then to the static state, rather than constant motion. Thus, studies on the development of the mechanism for landing or perching may have greater reference significance. Previously, Xu [17] developed a parallel-link-passive gripper for multicopters perching on plane-like targets by its own gravity, and researchers from University of Utah developed an avian-inspired passive mechanism for quadrotor perching [18, 19]. However, for some special occasions such as events succeeding a natural disaster, a multicopter is unable to find a plane or pipe-like object for perching or landing. Thus, the research on enabling multicopters to land on uneven ground or rough terrain is considered. A few researchers developed novel multicopters which can land on water [20] or even asteroid ground [21].

However, the aforementioned devices for landing utilize electrical power. These actuated landing devices need to be carried with power supply devices, such as power source or several batteries, and this increases the mass of the entire system, which may result in the multicopter overload. As illustrated in Fig. 1a, most of the previous researches regard landing as its only achievable function, which may limit the diversity of multicopter functions. Therefore, to make the multicopter not only land

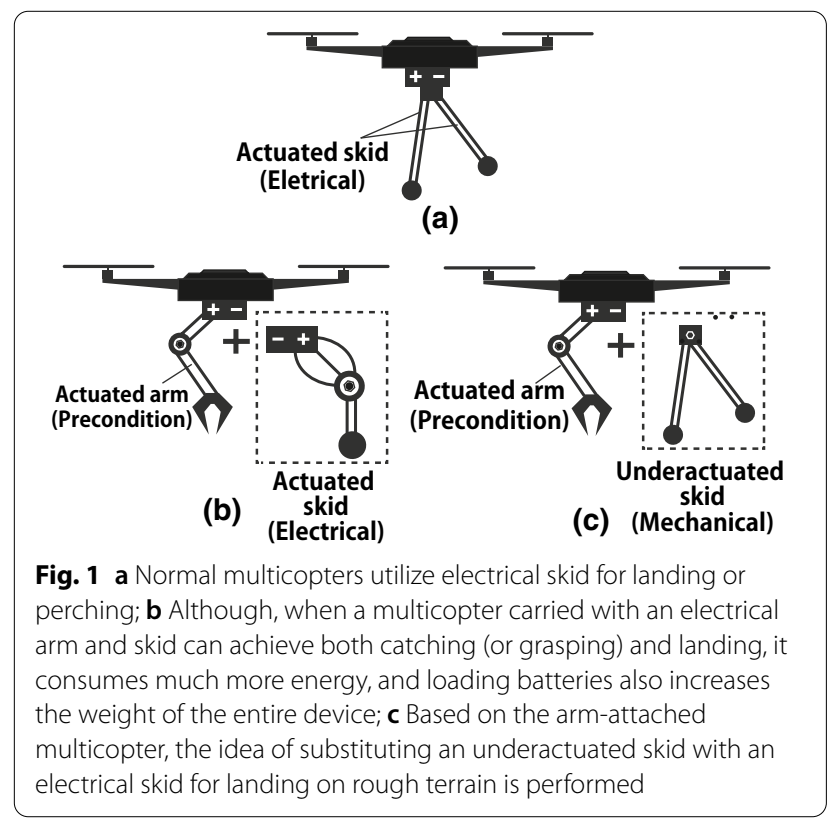

smoothly on a rough terrain, but also realize functions such as grasping, we proposed the device which is illustrated in Fig.1c [22]. In previous studies [22], although the structure of the landing device was proposed, for the proposed skid, we did not indicate how to determine whether it can land on a rough terrain or not. Therefore, in this study, we describe the judgment method of its landing possibility. In addition, considering the posture of the arm, the range of the uneven ground which can be landed is presented.

We organize the paper as follows: Section 2 describes the skid structure and the landing process. In section 3 we establish of the static model for analysis, and in section 4 , we present the anatomy of the static model and determine the range of available landing. Section 5 presents the verification experiment, and Section 6 presents the comparison of the results. Finally, in Section 7 we conclude the work and expound our future plans.

\section{Structure of the entire device and basic landing process}

Figure 2 illustrates the structural sketch of the entire proposed device, and it comprises of two parts, including the robot arm, multicopter body, and underactuated skid. In this study, to simplify the structure of the landing device, we proposed the robot arm, which utilizes a two-link manipulator and operates in a two-dimensional plane as presented in Fig. 2. The multicopter body in the middle is the main part of the device, and it carries the robot arm and the skid. The two legs on the right adopt a simple mechanical structure without attaching any other actuators. This is the most important characteristic because

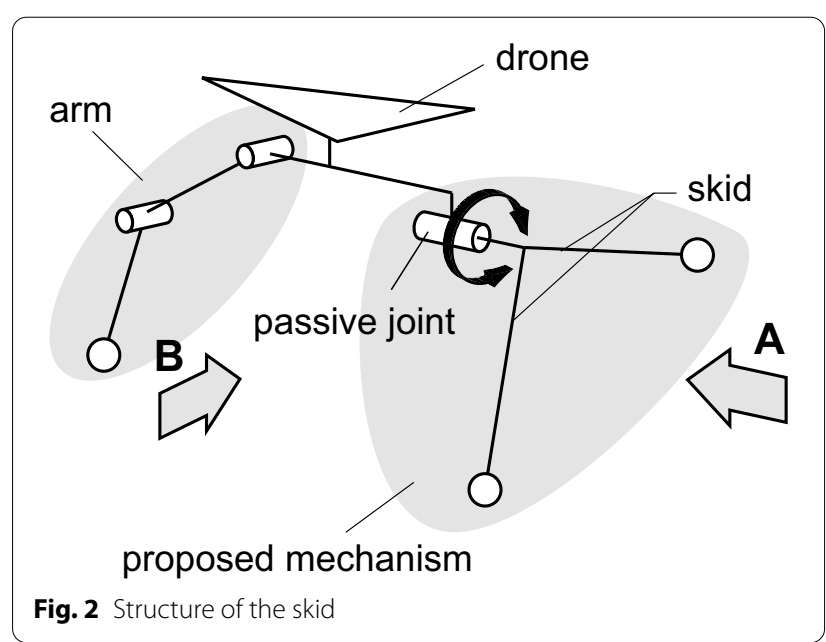


this proposal not only converses electric energy, but also reduces the overall weight of the multicopter.

The entire landing process is described in Figs. 3 and 4, from the two perspectives directions $A$ and $B$ illustrated in Fig. 2. Figure 3a illustrates the initial state when the multicopter is landing. Furthermore, as illustrated in Fig. 3b, one side of the foot contacts the ground first. While the entire device maintains its own horizontal position, the other side of the foot also contacts the ground because of the rotation of the passive joint (Fig. 3c). In Fig. 4a and b, the view is from the $B$ direction, which display the scenario after the two skids landed. By adopting the degree of freedom of the robot arm which can move on a vertical plane, all front-ends can be grounded, while making the entire device maintain its horizontal posture.

\section{Establishment of the static model}

To analyze the contact condition between the arm and the plane, we decomposed the device and established the static model as illustrated in Fig. 5. Because there is an inevitable occlusion relationship in the three-dimensional anatomical figure, we have utilized dotted lines to mark the occluded part. Similarly, auxiliary lines are also indicated by dashed lines.

Figure 5 reproduces the state of a landed device, but this time we concentrate on the robot arm model analysis. As illustrated in Fig. 5, we assume that there are three planes existing in the environment. Plane 1 denotes the lever plane, and also, to make the multicopter take off smoothly again, the multicopter must maintain its horizontal posture constantly. Thus, plane 1 is not only the lever plane, but also the plane where the body of multicopter is located. We define the direction of gravity as the direction of the $z$ axis, and plane 2 contains the axis of the passive joint and the normal vector of plane 1.Further, we define that the normal vector of plane 2 as $y$ axis and the $x$ axis can be obtained. The line of intersection is

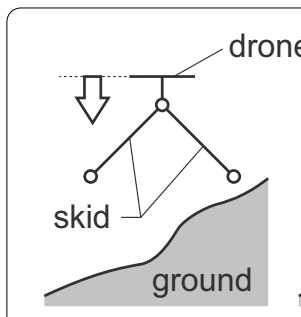

(a)

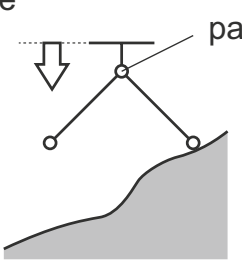

(b) passive joint

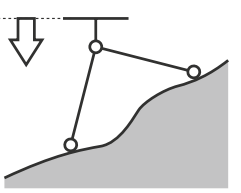

(c)
Fig. 3 Proposed skid landing flow as observed in direction $A$

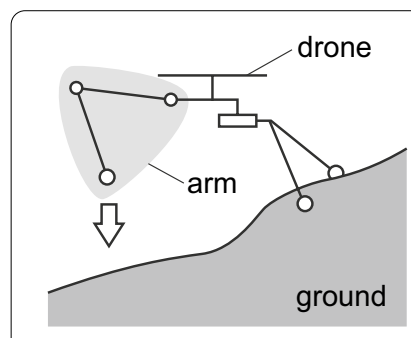

(a)

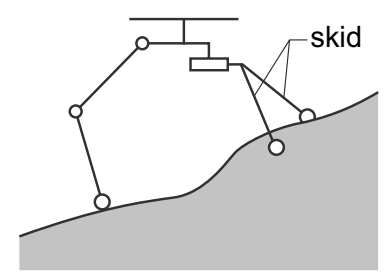

(b)
Fig. 4 Arm and landing flow as observed in direction $B$

denoted as $\boldsymbol{l}_{\boldsymbol{i}}$. We make a perpendicular line downward through the horizontal extension line of the passive joint $\boldsymbol{P}_{\mathbf{0 0}}$, and intersect the connection line of feet $\boldsymbol{P}_{\boldsymbol{r} \mathbf{1}}$ and $\boldsymbol{P}_{\boldsymbol{r} \mathbf{2}}$ at point $\boldsymbol{P}_{\boldsymbol{r}}$. Based on the origin $\boldsymbol{P}_{\boldsymbol{r}}$, the space rectangular coordinate system is established. Plane 3 is the plane for landing, the passive joint $\boldsymbol{P}_{\mathbf{0}} \mathbf{0}$, the position vectors, included $\boldsymbol{P}_{\mathbf{0}} \in R^{3}, \boldsymbol{P}_{\mathbf{1}} \in R^{3}$ and $\boldsymbol{P}_{\mathbf{2}} \in R^{3}$ on the arm are also on plane 2 . Two contact points of the passive skid on plane 3 are assumed as $\boldsymbol{P}_{\boldsymbol{r} \mathbf{1}}$ and $\boldsymbol{P}_{\boldsymbol{r} \mathbf{2}}$. Thus, including $\boldsymbol{P}_{\mathbf{2}}$, $\boldsymbol{P}_{\boldsymbol{r} \mathbf{1}}$ and $\boldsymbol{P}_{\boldsymbol{r} \mathbf{2}}$, there are three contact points on plane 3 and they are supporting the entire device.

\section{The capable range of slope for landing}

For landing on an uneven ground, it is necessary to know what level of ground can make the proposed device land successfully. As mentioned in the previous section, we established that plane 3 is the landing slope, and the most important characteristic, the gradient, will affect landing status. Therefore, the analysis of the capable landing range is given as follows.

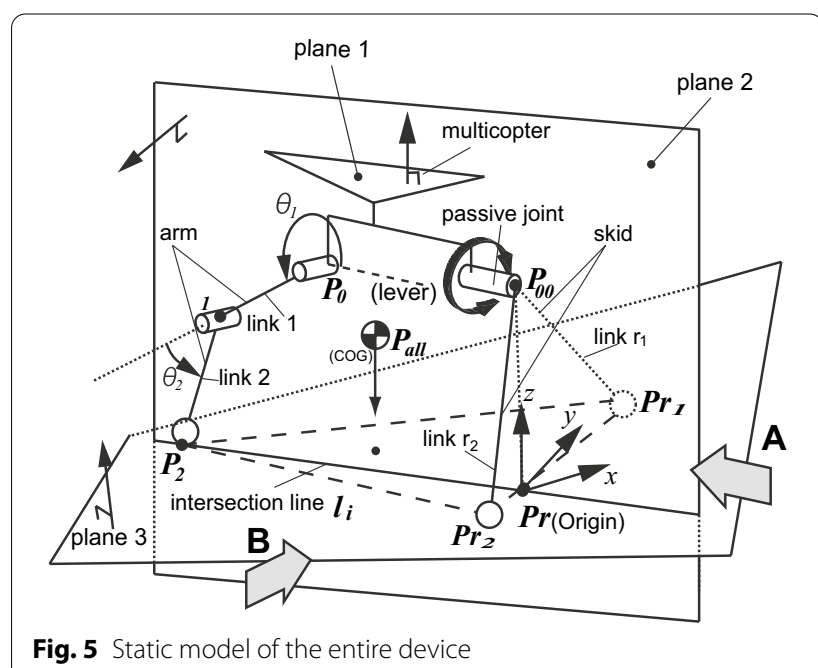




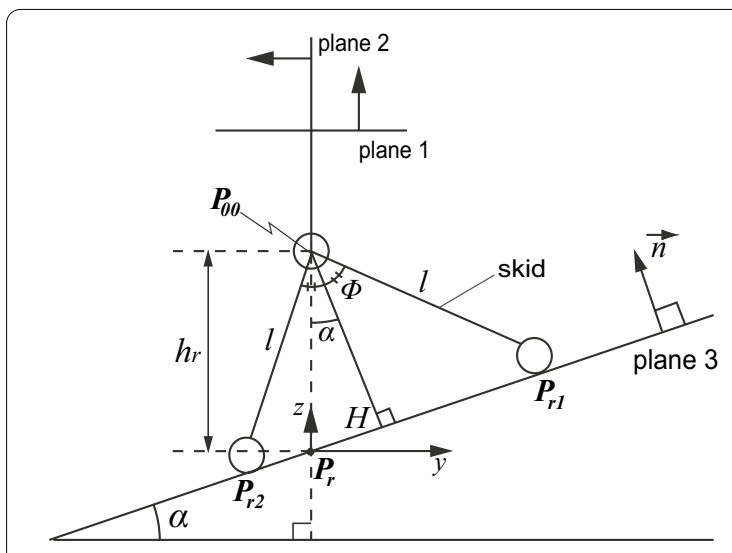

(a)

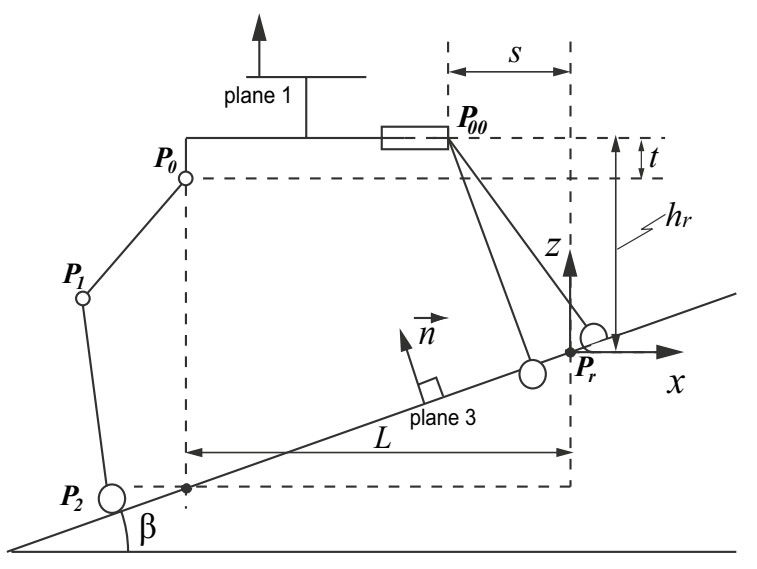

(b)

Fig. 6 View of the device from $A$ and $B$ directions

\section{Anatomy of the static model}

Figure $6 \mathrm{~A}$ and $\mathrm{B}$ are analytical graphs of Fig. 5 viewed from direction $\mathrm{A}$ (the view from $\mathrm{x}$ direction) and $\mathrm{B}$ (the view from $y$ direction). Aforementioned, plane 2 contains the axis of the passive joint and the normal vector of plane 1 , thus plane 2 can be expressed as $y=0$. We define the normal vector of plane 3 as $\boldsymbol{n}=\left[\begin{array}{lll}a & b & c\end{array}\right]^{t}$, and we obtain:

$$
a x+b y+c z=0 .
$$

We define that the inclination angle viewed from direction $\mathrm{A}$ is $\alpha$, and it can be obtained as:

$$
\alpha=-\tan ^{-1} \frac{b}{c}
$$

In Fig. 6A we illustrate that $\boldsymbol{H}$ is the intersection of plane 3 and the normal vector of plane 3 , which passes through $\boldsymbol{P}_{\mathbf{0 0}}$. Then the angle $\angle \boldsymbol{H P}_{\mathbf{0 0}} \boldsymbol{P}_{\boldsymbol{r} \mathbf{1}}$ is defined as $\phi$. We illustrate that the distance between $\boldsymbol{P}_{\mathbf{0 0}}$ and $\boldsymbol{P}_{\boldsymbol{r}}$ is $h_{r}$, and it can be obtained as:

$$
h_{r}=\frac{l \cos \phi}{\cos \alpha}
$$

Therefore the position vector of $\boldsymbol{P}_{\boldsymbol{r} 1}$ and $\boldsymbol{P}_{\boldsymbol{r} 2}$ can be obtained as:

$$
\begin{aligned}
& \boldsymbol{P}_{r 1}=\left(\begin{array}{l}
P_{r 1 x} \\
P_{r 1 y} \\
P_{r 1 z}
\end{array}\right)=\left(\begin{array}{l}
0 \\
\left(h_{r} \sin \alpha+\sin \phi\right) \cos \alpha \\
\left(h_{r} \sin \alpha+\sin \phi\right) \sin \alpha
\end{array}\right) \\
& \boldsymbol{P}_{r 2}=\left(\begin{array}{l}
P_{r 2 x} \\
P_{r 2 y} \\
P_{r 2 z}
\end{array}\right)=\left(\begin{array}{l}
0 \\
\left(l \sin \phi-h_{r} \sin \alpha\right) \cos (\pi+\alpha) \\
\left(l \sin \phi-h_{r} \sin \alpha\right) \sin (\pi+\alpha)
\end{array}\right)
\end{aligned}
$$

Figure $6 \mathrm{~B}$ is the analytical graph of Fig. 5 viewed from direction $B$ and similarly we can obtain that:

$$
\beta=-\tan ^{-1} \frac{a}{c}
$$

We denote that the horizontal and vertical distances between $\boldsymbol{P}_{\boldsymbol{r}}$ and $\boldsymbol{P}_{\mathbf{0 0}}$ are $s$ and $h_{r}$, respectively. Thus the position vector of $\boldsymbol{P}_{\mathbf{0 0}}$ can be obtained as:

$$
\boldsymbol{P}_{00}=\left(\begin{array}{l}
P_{00 x} \\
P_{00 y} \\
P_{00 z}
\end{array}\right)=\left(\begin{array}{l}
-s \\
0 \\
h_{r}
\end{array}\right)
$$

For the position vector of $\boldsymbol{P}_{\mathbf{0}}$, we denote that the horizontal distance between $\boldsymbol{P}_{\boldsymbol{r}}$ and $\boldsymbol{P}_{\mathbf{0}}$ is $L$, and the vertical distance between $\boldsymbol{P}_{\mathbf{0 0}}$ and $\boldsymbol{P}_{\mathbf{0}}$ is $t$. Therefore the position vector of $\boldsymbol{P}_{\mathbf{0}}$ can be obtained as:

$$
\boldsymbol{P}_{0}=\left(\begin{array}{l}
P_{0 x} \\
P_{0 y} \\
P_{0 z}
\end{array}\right)=\left(\begin{array}{l}
-L \\
0 \\
h_{r}-t
\end{array}\right)
$$

As illustrated in Fig. 5, the angle between link 1 and the lever is $\theta_{1}$, and the angle between link 2 and the extension line of link 1 is $\theta_{2}$. We assume that the length of link 1 and link 2 are $l_{1}$ and $l_{2}$. Therefore, $\boldsymbol{P}_{\mathbf{1}}$ and $\boldsymbol{P}_{\mathbf{2}}$ can be expressed as: 


$$
\begin{gathered}
\boldsymbol{P}_{\mathbf{1}}=\left(\begin{array}{l}
P_{1_{x}} \\
P_{1_{y}} \\
P_{1_{z}}
\end{array}\right)=\boldsymbol{P}_{\mathbf{0}}+\left(\begin{array}{l}
l_{1} \cos \theta_{1} \\
0 \\
l_{1} \sin \theta_{1}
\end{array}\right) \\
\boldsymbol{P}_{\mathbf{2}}=\left(\begin{array}{l}
P_{2_{x}} \\
P_{2_{y}} \\
P_{2_{z}}
\end{array}\right)=\boldsymbol{P}_{\mathbf{0}}+\left(\begin{array}{l}
l_{1} \cos \theta_{1}+l_{2} \cos \left(\theta_{1}+\theta_{2}\right) \\
0 \\
l_{1} \sin \theta_{1}+l_{2} \sin \left(\theta_{1}+\theta_{2}\right)
\end{array}\right)
\end{gathered}
$$

\section{Judgment of the ground-contacting state}

To assess the ground-contacting condition, contact points $\boldsymbol{P}_{\mathbf{2}}, \boldsymbol{P}_{\boldsymbol{r} \mathbf{1}}$ and $\boldsymbol{P}_{\boldsymbol{r} \mathbf{2}}$ are considered, and the view is from the $y$ direction. Hence, the plane 3 and the device are simplified as points and lines. The intersection of plane 2 and plane 3 is:

$$
a x+c z=0
$$

$\boldsymbol{P}_{2}$ is on that line, thus we obtain

$$
a P_{2 x}+c P_{2 z}=0
$$

When $\theta_{2}$ is a constant, and if link 1 rotates around $P_{0}$, the trajectory of $\boldsymbol{P}_{\mathbf{2}}$ is a circle, and the distance from $\boldsymbol{P}_{\mathbf{2}}$ to $\boldsymbol{P}_{\mathbf{0}}$ is the radius $r$. We define the functional expression of the circle as:

$$
\left(P_{2 x}-P_{0 x}\right)^{2}+\left(P_{2 z}-P_{0 z}\right)^{2}-r^{2}=0
$$

By simultaneous Eqs. (12) and (13), and to concretize $P_{0 x}$ and $P_{0 z}$ by Eq. (8), we obtained

$$
A P_{2 x}^{2}+B P_{2 x}+C=0
$$

where:

$$
\begin{aligned}
& A=1+\frac{a^{2}}{c^{2}} \\
& B=\frac{2 a\left(h_{r}-t\right)-2 L}{c} \\
& C=-L^{2}+.\left(h_{r}-t\right)^{2}-r^{2}
\end{aligned}
$$

and the discriminant as:

$$
\Delta=B^{2}-4 A C
$$

As illustrated in Fig. 7, for the discriminant $\Delta$, if the condition $\Delta \geq 0$ can be satisfied, we can conclude that point $\boldsymbol{P}_{\mathbf{2}}$ and plane 3 have at least one intersection.

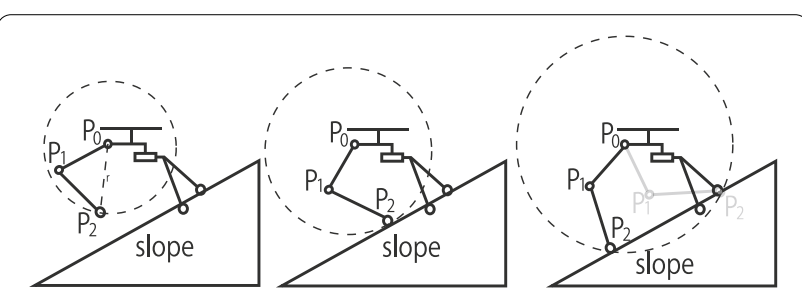

(a) $\Delta<0$

(b) $\Delta=0$

(c) $\Delta>0$

\section{Condition of non-tilting}

The entire device can be divided into four parts: the Part 1 is the link 1, the Part 2 is the link 2 and the multicopter body can be defined as the Part 3 .The skid, which comprises link $l_{r 1}$, link $l_{r 2}$ and the passive joint, is defined as the Part 4 . The position vectors of the COG of Part $i(i=1,2,3,4)$ are defined as $\boldsymbol{P}_{\boldsymbol{a} i}(\mathrm{i}=1,2,3,4)$. We define that the mass of each part as $m_{i},(i=1,2, \ldots 4)$, and then the COG of each part can be obtained. Then the COG of the entire device can be obtained as:

$$
P_{\text {all }}=P_{\text {allx }} P_{\text {ally }}=\frac{\frac{\sum_{i=1}^{4} m_{i} x_{a} i}{\sum_{i=1}^{4} m_{a} i}}{P_{i=1}^{4} y_{a} i}
$$

As illustrated in Fig. 5, the contact points $\boldsymbol{P}_{\mathbf{2}}, \boldsymbol{P}_{\boldsymbol{r} \mathbf{1}}$ and $\boldsymbol{P}_{\boldsymbol{r} \mathbf{2}}$ form a triangle located on plane 3 . Thus, if the projection of the COG of the entire device can remain inside the triangle consisting of $\boldsymbol{P}_{\mathbf{2}} \boldsymbol{P}_{\boldsymbol{r} \mathbf{1}} \boldsymbol{P}_{\boldsymbol{r} \mathbf{2}}$, the skid-driven multicopter can remain balanced. If the COG stays outside the triangle, the multicopter will tilt.

In this analysis, it is only necessary to consider its projection on the $x-y$ plane. Therefore, we define that $\boldsymbol{p}_{\text {all }}$ as COG coordinate of the entire device on $x-y$ plane, and we define:

$$
p_{\text {all }}=\frac{P_{\text {all }}}{P_{\text {all }}}
$$

The projection of $\boldsymbol{P}_{\boldsymbol{r} \mathbf{1}}, \boldsymbol{P}_{\boldsymbol{r} \mathbf{2}}$, and $\boldsymbol{P}_{\mathbf{2}}$ are onto the $x-y$ plane respectively, we define:

$$
\begin{aligned}
& \boldsymbol{p}_{\boldsymbol{r} \mathbf{1}}=\left(\begin{array}{l}
P_{r 1_{x}} \\
P_{r 1_{y}}
\end{array}\right) \\
& \boldsymbol{p}_{\boldsymbol{r} \mathbf{2}}=\left(\begin{array}{c}
P_{r 2_{x}} \\
P_{r 2_{y}}
\end{array}\right)
\end{aligned}
$$




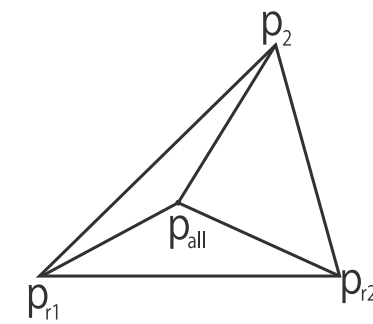

(a) $p_{\text {all }}$ is inside $\Delta p_{r 1} p_{12} p_{2}$

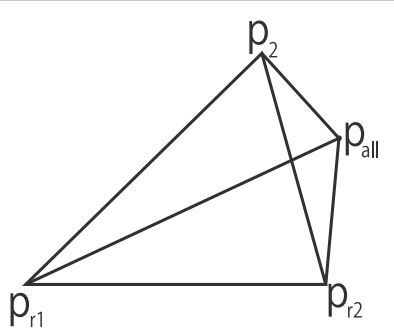

(b) $p_{\text {all }}$ is outside $\Delta p_{\mathrm{r}} p_{12} p_{2}$

Table 1 Properties of items of the entire system

\begin{tabular}{lll}
\hline Mass & $m_{1}$ & $0.24 \mathrm{~kg}$ \\
& $m_{2}$ & $0.24 \mathrm{~kg}$ \\
& $m_{3}$ & $0.88 \mathrm{~kg}$ \\
Length & $m_{4}$ & $0.086 \mathrm{~kg}$ \\
& $L$ & $205 \mathrm{~mm}$ \\
& $I$ & $300 \mathrm{~mm}$ \\
& $I_{1}$ & $150 \mathrm{~mm}$ \\
& $I_{2}$ & $150 \mathrm{~mm}$ \\
& $s$ & $60 \mathrm{~mm}$ \\
Angle & $t$ & $40 \mathrm{~mm}$ \\
& $v$ & $150 \mathrm{~mm}$ \\
& $w$ & $150 \mathrm{~mm}$ \\
& $\phi$ & $62\left(^{\circ}\right)$ \\
$\theta_{1 \min }$ & $135\left(^{\circ}\right)$ \\
$\theta_{1 \max }$ & $270\left(^{\circ}\right)$ \\
$\theta_{2 \min }$ & $0\left(^{\circ}\right)$ \\
$\theta_{2 \max }$ & $180\left(^{\circ}\right)$ \\
$g$ & $9.80 \mathrm{~m} / \mathrm{s}^{2}$ \\
\hline
\end{tabular}

$$
\boldsymbol{p}_{2}=\left(\begin{array}{c}
P_{2_{x}} \\
P_{2_{y}}
\end{array}\right)
$$

As illustrated in Fig. 8, when the projection of $\boldsymbol{P}_{\text {all }}$ can remain in the range of $\triangle \boldsymbol{P}_{\boldsymbol{r} \mathbf{1}} \boldsymbol{P}_{\boldsymbol{r} \mathbf{2}} \boldsymbol{P}_{\mathbf{2}}$ and we connect it to the three vertices of the triangle, three new triangles: $\triangle \boldsymbol{p}_{r 1} p_{2} p_{\text {all }}, \triangle \boldsymbol{p}_{r 2} \boldsymbol{p}_{2} \boldsymbol{p}_{\text {all }}$, and $\triangle \boldsymbol{p}_{r 1} \boldsymbol{p}_{\boldsymbol{r} 2} \boldsymbol{p}_{\text {all }}$ are obtained. Here we define that the area of $\triangle \boldsymbol{P}_{\boldsymbol{r} 1} \boldsymbol{P}_{\boldsymbol{r} 2} \boldsymbol{P}_{2}, \triangle \boldsymbol{p}_{\boldsymbol{r} 1} \boldsymbol{p}_{\mathbf{2}} \boldsymbol{p}_{\text {all }}$, $\triangle \boldsymbol{p}_{r 2} \boldsymbol{p}_{2} \boldsymbol{p}_{\text {all }}$, and $\triangle \boldsymbol{p}_{r 1} \boldsymbol{p}_{r 2} \boldsymbol{p}_{\text {all }}$ are $S \triangle \boldsymbol{P}_{r 1} \boldsymbol{P}_{r 2} \boldsymbol{P}_{2}, S \triangle \boldsymbol{p}_{r 1} \boldsymbol{p}_{2}$ $\boldsymbol{p}_{\text {all }}, S \triangle \boldsymbol{p}_{\boldsymbol{r} 2} \boldsymbol{p}_{2} \boldsymbol{p}_{\text {all }}$, and $S \triangle \boldsymbol{p}_{\boldsymbol{r} 1} \boldsymbol{p}_{\boldsymbol{r} 2} \boldsymbol{p}_{\text {all }}$. If $\boldsymbol{p}_{\text {all }}$ can remain in $\triangle \boldsymbol{P}_{\boldsymbol{r} 1} \boldsymbol{P}_{\boldsymbol{r} 2} \boldsymbol{P}_{2}$, we obtain:

$$
\begin{aligned}
S \triangle \boldsymbol{P}_{r 1} \boldsymbol{P}_{r 2} \boldsymbol{P}_{2} & =S \triangle \boldsymbol{p}_{r 1} \boldsymbol{p}_{2} \boldsymbol{p}_{\text {all }}+S \triangle \boldsymbol{p}_{\boldsymbol{r} 2} \boldsymbol{p}_{2} \boldsymbol{p}_{\text {all }} \\
& +S \triangle \boldsymbol{p}_{\boldsymbol{r} 1} \boldsymbol{p}_{\boldsymbol{r} 2} \boldsymbol{p}_{\text {all }}
\end{aligned}
$$

Therefore from Eq. (24) we can determine whether $\boldsymbol{p}_{\text {all }}$ remains inside or outside $\Delta \boldsymbol{P}_{\boldsymbol{r} \mathbf{1}} \boldsymbol{P}_{\boldsymbol{r} \mathbf{2}} \boldsymbol{P}_{\mathbf{2}}$, and we can also

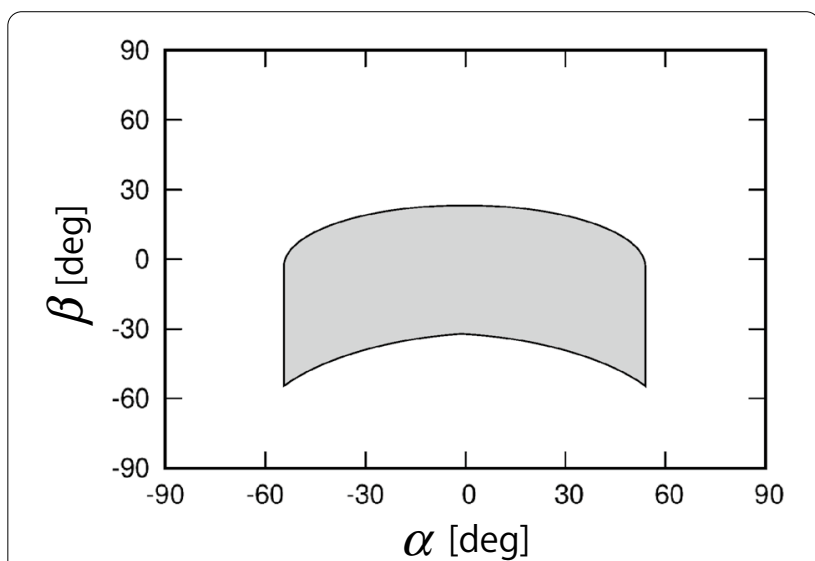

Fig. 9 Range of $\alpha$ and $\beta$ under landable conditions

determine whether the entire device can maintain its horizontal posture without tilting. As for where the $\boldsymbol{p}_{\text {all }}$ in the triangle can make the device the most stable, we consider it as one of the study in the future.

\section{Numerical analysis and theoretical value}

Table 1 presents the properties of items in the entire system. For an arbitrary slope, whether the multicopter can achieve stable landing or not, can be determined from Eqs.(18) and (24). By changing $\alpha$ and $\beta$, and using Eqs. (18) and (24), the range of $\alpha$ and $\beta$ on the slope that can be landed is obtained and it is summarized in Fig. 9. Fig. 9 illustrates the feasible range of the inclination angles $\alpha$ and $\beta$. The range of $\alpha$ is less than $\pm 60^{\circ}$, the maximum value of $\beta$ is approximately $20^{\circ}$ and the minimum value is approximately $-60^{\circ}$.

Furthermore, the relationship of the inclination angle $\alpha, \beta$ and $\theta_{1}, \theta_{2}$ when landing is achievable is summarized in Fig. 10. In Fig. 10, the value of $\theta_{1}$ is listed from $135^{\circ}$ to $270^{\circ}$, with an interval of $15^{\circ}$.

\section{Experimental setting}

The aim of the experiment is to verify that by changing the angle of links and other conditions in actual environment, the entire device can maintain its horizontal posture within the range concluded in the previous sections. By evaluating the performance of the proposed device while landing on a slope with different inclination angles, we aim to verify the consistency of actual experimental and theoretical result.

\section{Experimental environment}

Figure 11 illustrates the experimental environment. We chose a board with the length of $1200 \mathrm{~mm}$ and width of 
$850 \mathrm{~mm}$ as the slope for the experiment. Several bricks were utilized to support the board at its four corners. By increasing or decreasing the number of bricks needed to alter the supported height of the board, and different inclination angles $\alpha$ and $\beta$ were obtained.

\section{prototype}

Figure 12 illustrates the prototype of the entire device. It can be divided into three main parts: from left to behind, the arm, multicopter body and proposed skid are shown. For the multicopter, we selected DJI FlameWheel450 [23] as the carrier. The arm is equipped with RC servo motors at the joints.

\section{Results}

\section{Experimental result of arbitrary area}

It is difficult to conduct the experiment of the entire range illustrated in Fig. 9, because there are too many combinations of $\alpha$ and $\beta$. Therefore, we consider that $\theta_{1}$ is a constant of $180^{\circ}$. The calculation of the range for available landing is summarized, and the experiment is conducted in that range. The range for experiment is illustrated in Fig. 13. Simultaneously, the range of $\alpha$ is approximately $0^{\circ}$ to $30^{\circ}$, and the range of $\beta$ is $2^{\circ}$ to $-18^{\circ}$. Then we set $\alpha$ to $0^{\circ}, 10^{\circ}, 20^{\circ}$, and $30^{\circ}$ degrees, and converted $\beta$ from $2^{\circ}$ to $-18^{\circ}$ in $2^{\circ}$ increments to verify whether landing was possible. The result of the verification is illustrated in Fig. 14, and for all cases can achieve stable landing is shown.

Figure 15 illustrates an enlarged view of the landing range in Fig. 14. In Fig. 15, the pattern in hollow circle $(\bigcirc)$ implies the cases that stable landing can be achieved, and the pattern in cross $(x)$ implies the cases of unavailable landing. The landing range and the experimental results are similar. This means that it is possible to determine whether landing is possible or not, by the method presented in the previous sections.

\section{Experimental result of area near the border}

As shown in Fig. 16, to make the analysis and conclusions more convincing, the experiment for the area near the border in Fig. 9 is also conducted. Because the range of $\alpha$ and $\beta$ for available landing in Fig. 9 is a symmetrical arc area, we take the highest and lowest points on the leftmost side of the range for the verification experiment. The point on the upper left is defined as point $A$ and the point on the bottom left is defined as point $B$. The coordinates of point $A$ and $B$ are $(-53,-6)$ and $(-53,-50)$ respectively, and an experimental snap shot is shown in Fig. 17.
Figure 17 shows that the device can maintain its horizontal state in the case when the inclination angles are at the point $\mathrm{A}$ and $\mathrm{B}$ positions. When in the point $\mathrm{A}$ coordinate position, the device can maintain its stable state only when the angles of $\theta_{1}$ and $\theta_{2}$ are $220^{\circ}$ and $20^{\circ}$ respectively, and also, when in the point $\mathrm{B}$ coordinate position, the device can maintain its stable state only when the angles of $\theta_{1}$ and $\theta_{2}$ are $140^{\circ}$ and $165^{\circ}$, respectively.

\section{Discussion}

Analysis of the landing conditions implicated that the feasible range for multicopter landing, and theoretically, we can determine the possibility of landing on a plane with different inclination angles according to the proposed judgment method. However, the analysis at this stage still has some insufficient. One of the limitation is that although the wooden board are utilized to simulate inclined plane in our experiment for simplicity, the actual environment, especially after a disaster, is often much more complicated, and its analysis is considered to be improved.

Based on the results of the theoretical and experimental values, the error in analysis is also considered. In this study, two reasons are considered to be the factor of such errors: (1) First is the error in judgment of the COG. Each part of the multicopters COG can be analyzed by hanging, (Plumb Line Technique) and this may cause the error when calculating the COG of the entire device, and also affect determining the available landing; (2) The second possible error is in angle measurement. Although the skid and arm are considered as a single link in static model analysis, they are structures with thickness. When conducting experiments, we measure the angular relationship based on the central axis of these links, which may lead to a few slight errors.

Additionally, the analysis of the contact point to the landing plane is also considered. Although for the same slope, there are many contact points that can make the device landing smoothly, there is an optimal location of the $\boldsymbol{P}_{\text {all }}$ and the contact point. As shown in Fig. 18, if we define three sides of the triangle $\boldsymbol{P}_{\mathbf{2}} \boldsymbol{P}_{\boldsymbol{r} \mathbf{1}} \boldsymbol{P}_{\boldsymbol{r} \mathbf{2}}$ are $a, b$ and $c$. Then the distant between the projection of $\boldsymbol{P}_{\text {all }}$ and those three sides, are defined as $l_{a}, l_{b}$, and $l_{c}$. If the shortest distant of $l_{a}, l_{b}$, and $l_{c}$ can be as long as possible, the stability of the device in this case can be considered as the best. As for the location of the contact point under different conditions, when the entire device is the most stable, it is considered as one of the future work.

(See figure on next page.)

Fig. 10 The relationship of the inclination angle $\alpha, \beta$ and the angle of robot $\operatorname{arm} \theta_{1}, \theta_{2}$ when landing is achievable 

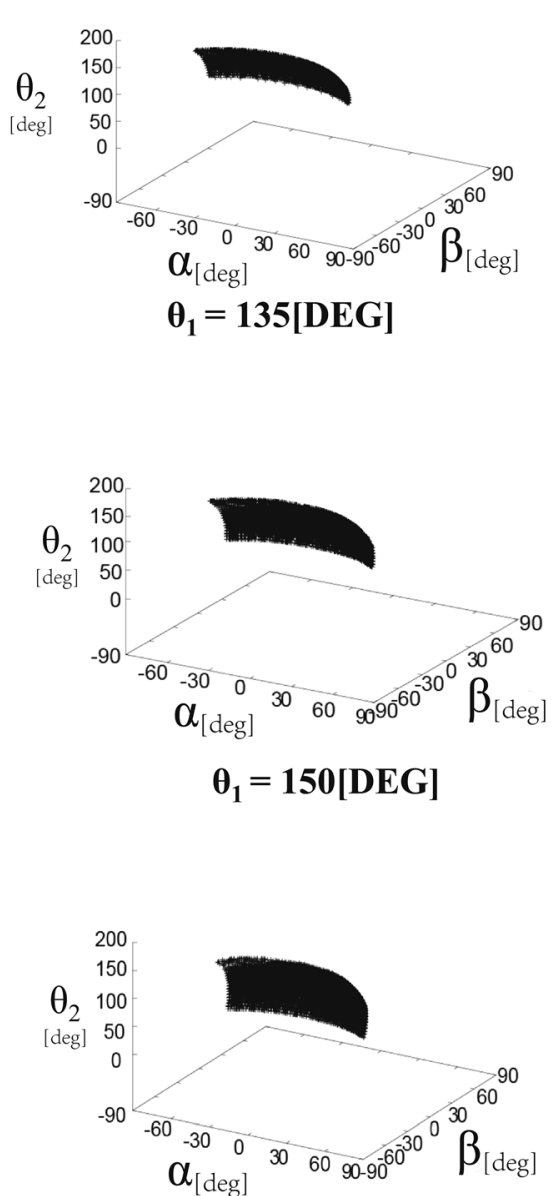

$$
\theta_{1}=165[\mathrm{DEG}]
$$
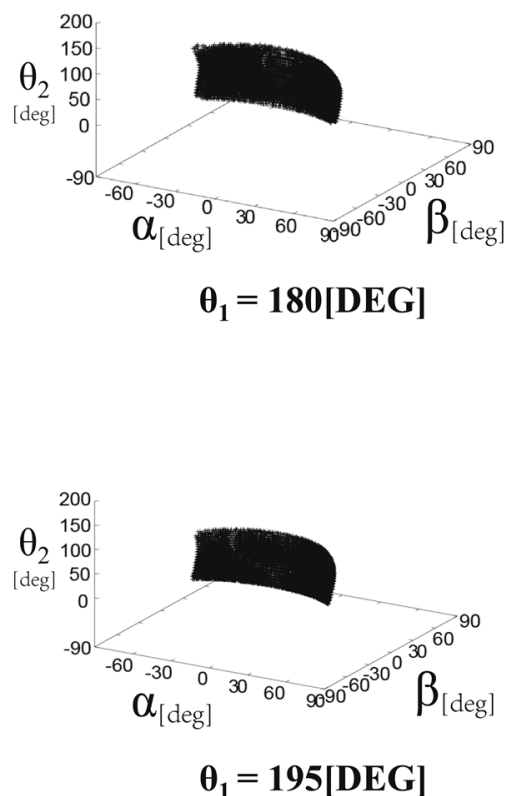
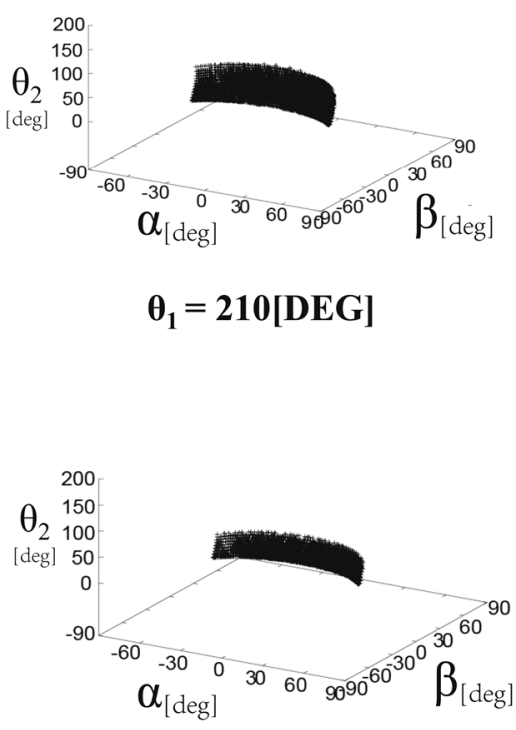

$\theta_{1}=225[\mathrm{DEG}]$

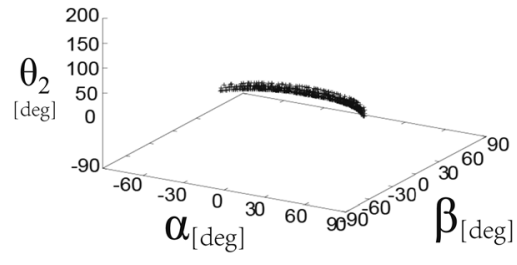

$\theta_{1}=240[\mathrm{DEG}]$
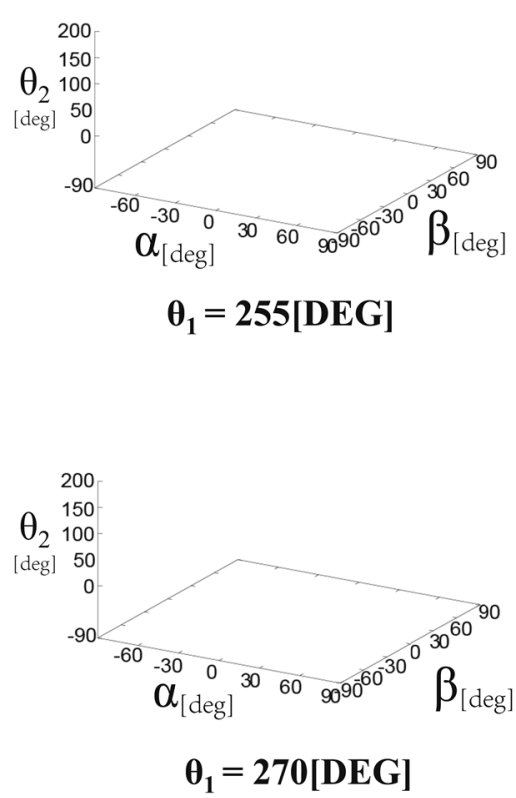

Fig. 10 (See legend on previous page.) 


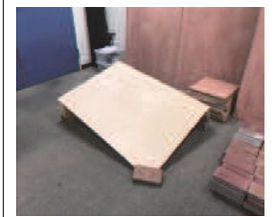

(a)

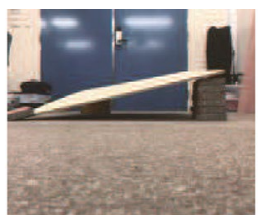

(b)

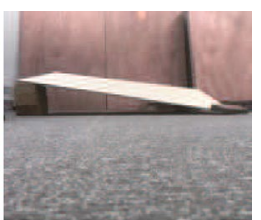

(c)
Fig. 11 The Experimental environment. $\mathbf{a}$ is the overview of the experimental environment. $\mathbf{b}$ and $\mathbf{c}$ are the view from $\mathrm{x}$ and $\mathrm{y}$ direction of the environment respectively

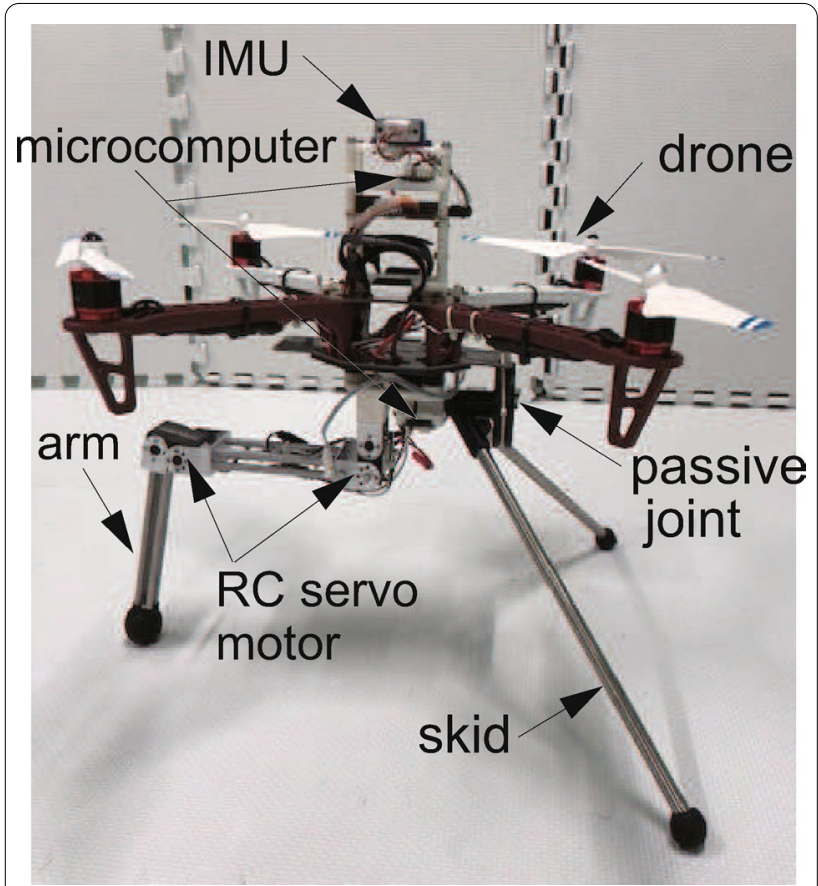

Fig. 12 The proposed device prototype

\section{Conclusion}

In this study, we proposed a method to determine whether or not the skid-carried multicopter can land on an uneven ground. The slope range where the multicopter can land is concluded, and the accuracy of the analysis is demonstrated by the verification experiment. However, some weaknesses and improvements also need to be considered in our study. For example, to simplify the analysis, we simulated the uneven ground with slopes with different inclination angles, and this may not a perfect explanation of the correspondence between the device and the rough terrain. Therefore, in the next stage, we consider

\section{Enlarged view of the sample area}

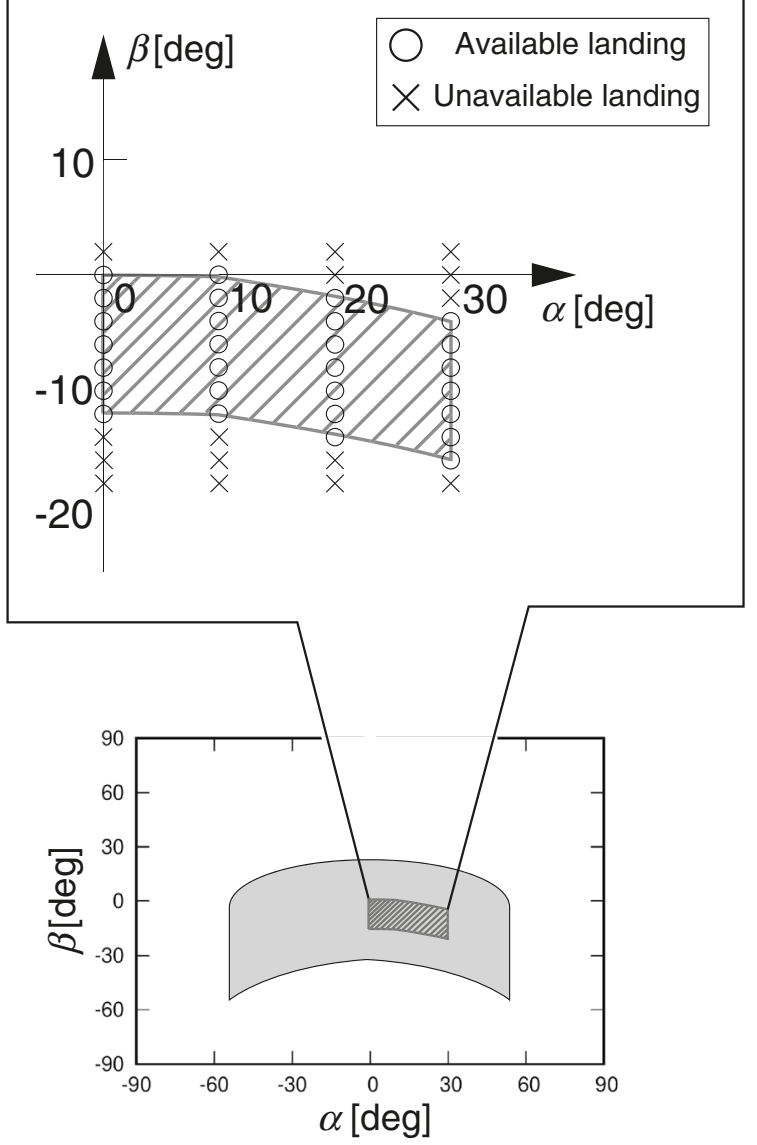

Fig. 13 The selected sample area for experiment

utilizing an environment with obstacles to simulate uneven ground to make the research closer to reality.

In addition, when considering the coefficient of friction, we solely selected rubber and wood as research objects, and this may cause several study limitations. Thus, in the future, we plan to analyze the possibility of landing, when the surface of the slope is made of other materials. We also planned to explore how the length will affect the experimental results. If the possibility of landing can be controlled by changing the link length, it would be a very interesting and meaningful study. In addition, we anticipate improving the procedure of the flight controller, and achieving outdoor landings. 


\section{$a=0$ [deg]}

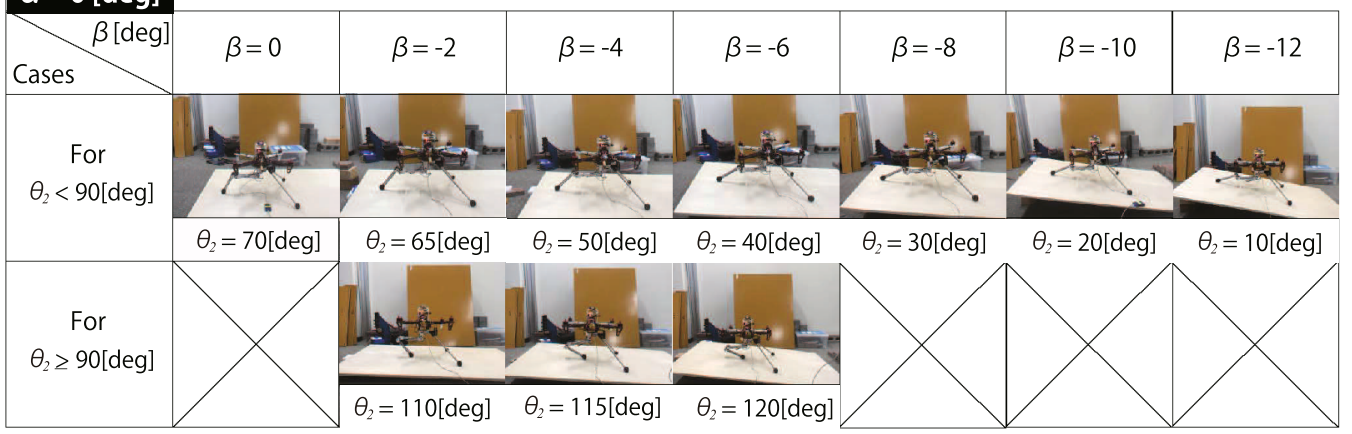

\section{$a=10$ [deg]}

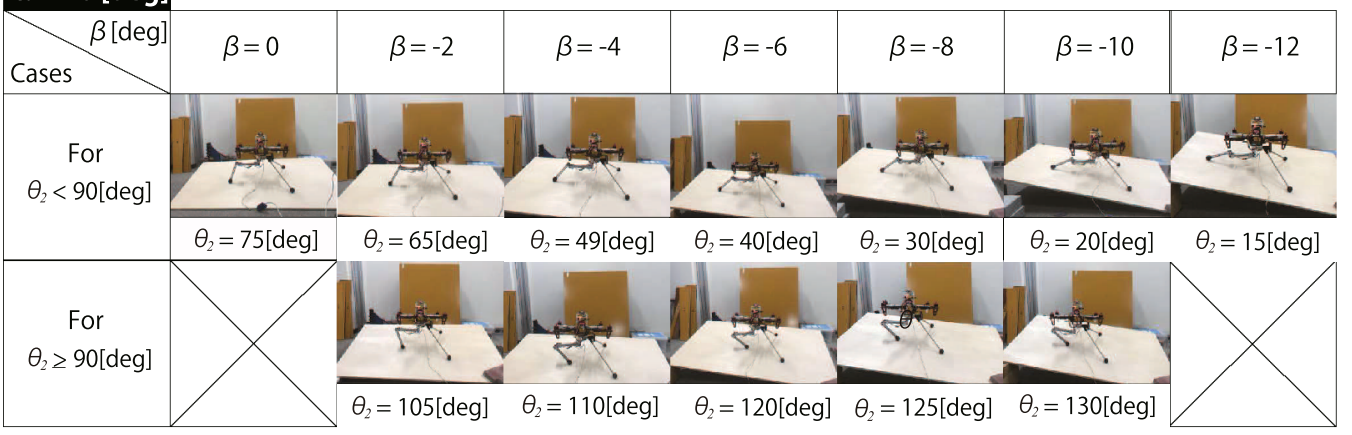

\section{$a=20$ [deg]}

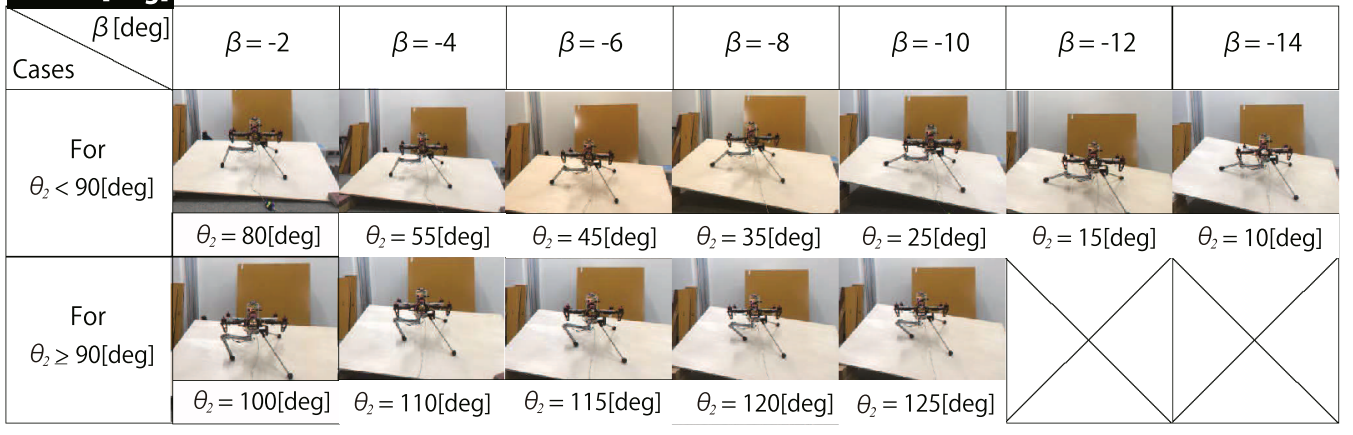

\section{$a=30$ [deg]}

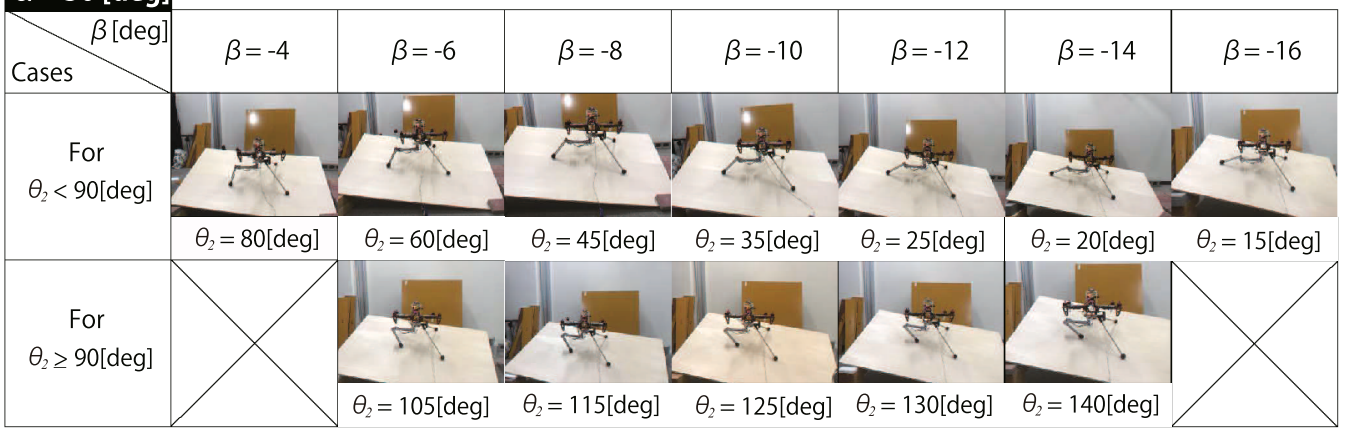

Fig. 14 Snap shots of the maintained horizontal state experiment 


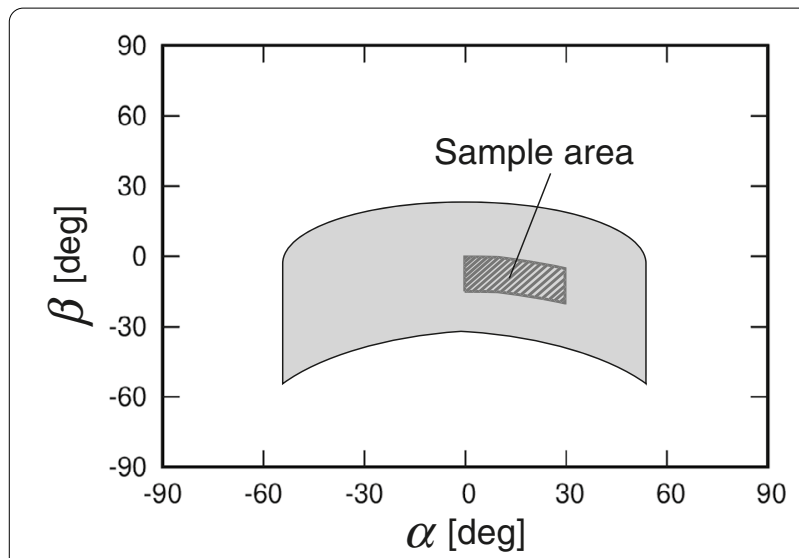

Fig. 15 The enlarged view of the sample area's landing range illustrated in Fig. 15

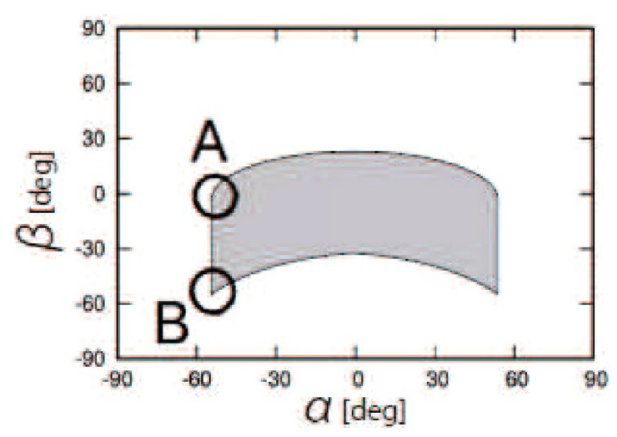

Fig. 16 The selected points near the border for experiment

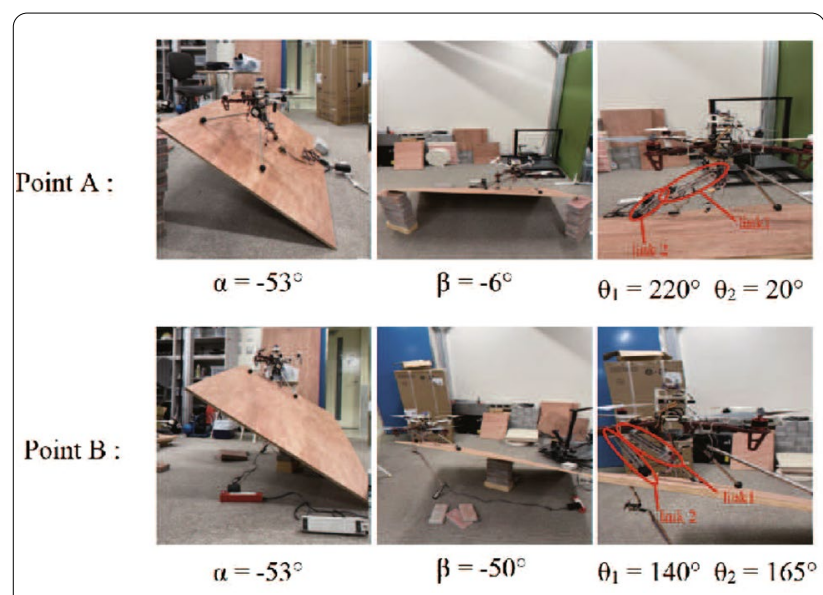

Fig. 17 Experiment of the area near the border in Fig. 9

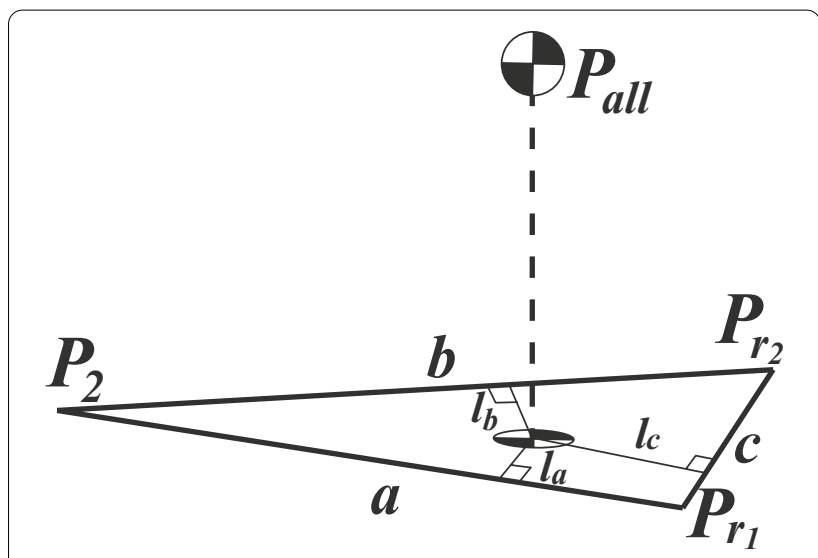

Fig. 18 Explanation for the optimal location of the $\boldsymbol{P}_{\text {all }}$ and the contact point

\section{Acknowledgements}

The authors would like to acknowledge the support of the Smart Robotics Laboratory, Graduate School of Engineering, Hiroshima University in Japan for their contributions.

\section{Authors' contributions}

MX and TT carried out the concept, design, and experiment of this study. MX drafted the manuscript, and TT and TS revised and refined the manuscript. All authors read and approved the final manuscript.

\section{Funding}

Not applicable.

\section{Availability of data and materials}

The analysis and experimental results during the current research are available from the corresponding author on reasonable request.

\section{Declarations}

\section{Competing interests}

The authors declare that they have no competing interests.

Received: 18 August 2021 Accepted: 17 October 2021

Published online: 22 November 2021

\section{References}

1. Appeaning Addo K, Jayson-Quashigah PN, Codjoe SNA, Martey F (2018) Drone as a tool for coastal flood monitoring in the Volta Delta, Ghana. Geoenviron Disasters 5(1):1-3

2. Patrik A et al (2019) GNSS-based navigation systems of autonomous drone for delivering items. J Big Data 6(1):1-4

3. Ohnishi Yoshinori, Takaki Takeshi, Aoyama Tadayoshi, Ishii Idaku (2017) Development of a 4-Joint 3-DOF Robotic Arm with Anti-reaction Force Mechanism for a Multicopter, Proceeedings of 2017 IEEE/RSJ International Conference on Intelligent Robots and Systems, pp 985-991

4. Nagarjuna K (2015) Nagarjuna K, Suresh GR (2015) Design of effective landing mechanism for fully autonomous Unmanned Aerial Vehicle, 2015 3rd Int. Conf. Signal Process. Commun. Networking, ICSCN 2015 
5. Chandra KR, Ghosh S (2019) Hu-moment-based autonomous landing of a UAV on a hemispherical dome, 2019 Int. Conf. Unmanned Aircr. Syst. ICUAS 2019 2(1):19-25

6. Lan G, Bu Y, Liang J, Hao Q (2016) Action synchronization between human and UAV robotic arms for remote operation. 2016 IEEE Int. Conf. Mechatronics Autom. IEEE ICMA 2016:2477-2481

7. Ruggiero F, et al. (2015) A multilayer control for multirotor UAVs equipped with a servo robot arm, Proc.-IEEE Int. Conf. Robot. Autom., vol. 2015-June, no. June, pp 4014-4020

8. Bazylev D, Kremlev A, Margun A, Zimenko K (2016) Design of control system for a four-rotor UAV equipped with robotic arm, Int. Congr. Ultra Mod. Telecommun. Control Syst. Work., vol. 2016-January, pp. 144-149

9. Yamauchi G, Nagatani K, Hashimoto T, Fujino K (2017) Slip-compensated odometry for tracked vehicle on loose and weak slope. Robomech J. $4(1): 1-11$

10. Jayasekara PG, Arisumi H (2015) Surmounting obstacles by arm maneuver for unmanned power shovel. Robomech J. 2(1):1-12

11. Chen K et al (2018) Compound locomotion control system combining crawling and walking for multi-crawler multi-arm robot to adapt unstructured and unknown terrain. Robomech J. 5(1):1-7

12. Endo D, Nagatani K (2016) Assessment of a tracked vehicle's ability to traverse stairs. Robomech J. 3(1):1-13

13. Nakajima S (2020) Stair-climbing gait for a four-wheeled vehicle. Robomech J. 7(1):1-8

14. Wardana Ananta Adhi, Takaki Takeshi, Jiang Mingjun, Ishii Idaku (2020) Development of a single-wheeled inverted pendulum robot capable of climbing stairs. Adv Robot 34(10):674-688

15. Ohara K et al (2018) Energy-efficient narrow wall climbing of six-legged robot. Robomech J. 5(1):1

16. Yamamoto K, Aoki T (2020) Development of novel mobile robot with semicircular wheels. Robomech J. 7(1):1-11
17. Xu Maozheng, Takaki Takeshi, Jiang Mingjun, Ishii Idaku (2019) Development of Parallel-link-passive-gripper by Using a Multicopter's Own Weight for Perching, Proceedings of the SICE Annual Conference 2019, pp 431-432

18. Doyle CE et al (2013) An avian-inspired passive mechanism for quadrotor perching. IEEE/ASME Trans Mechatron 18(2):506-517. https://doi.org/10. 1109/TMECH.2012.2211081

19. Doyle CE, et al. (2011) Avian-inspired passive perching mechanism for robotic rotorcraft, 2011 IEEE/RSJ International Conference on Intelligent Robots and Systems, San Francisco, CA, pp 4975-4980. https://doi.org/10. 1109/IROS.2011.6094487

20. Bai Y, Hong JH, Zolotas A (2019) Development of a Novel Water Landing UAV with Deflatable Floater, 2019 Int. Work. Res. Educ. Dev. Unmanned Aer. Syst. RED-UAS 2019, pp 166-171

21. Zhao Z, Zhao J, Liu H (2012) An asteroid landing mechanism and its landing simulation, 2012 IEEE Int. Conf. Robot. Biomimetics, ROBIO 2012 Conf. Dig., pp 20-210

22. Xu Maozheng, Sumida Naoto, Takaki Takeshi (2020) Development of a passive skid for multicopter landing on Rough Terrain, Proceedings of IEEE/RSJ International Conference on Intelligent Robots and Systems, pp $1316-1321$

23. http://dl.djicdn.com/downloads/flamewheel/en/F550_User_Manual_ v2.0_en.pdf

\section{Publisher's Note}

Springer Nature remains neutral with regard to jurisdictional claims in published maps and institutional affiliations.

\section{Submit your manuscript to a SpringerOpen ${ }^{\circ}$ journal and benefit from:}

- Convenient online submission

- Rigorous peer review

- Open access: articles freely available online

- High visibility within the field

- Retaining the copyright to your article

Submit your next manuscript at $\boldsymbol{\nabla}$ springeropen.com 